\title{
THE FIGHTER PROBLEM: OPTIMAL ALLOCATION OF A DISCRETE COMMODITY
}

\author{
JAY BARTROFF, ${ }^{*}$ University of Southern California \\ ESTER SAMUEL-CAHN, ${ }^{* *}$ The Hebrew University of Jerusalem
}

\begin{abstract}
In this paper we study the fighter problem with discrete ammunition. An aircraft (fighter) equipped with $n$ anti-aircraft missiles is intercepted by enemy airplanes, the appearance of which follows a homogeneous Poisson process with known intensity. If $j$ of the $n$ missiles are spent at an encounter, they destroy an enemy plane with probability $a(j)$, where $a(0)=0$ and $\{a(j)\}$ is a known, strictly increasing concave sequence, e.g. $a(j)=$ $1-q^{j}, 0<q<1$. If the enemy is not destroyed, the enemy shoots the fighter down with known probability $1-u$, where $0 \leq u \leq 1$. The goal of the fighter is to shoot down as many enemy airplanes as possible during a given time period [0, $T]$. Let $K(n, t)$ be the smallest optimal number of missiles to be used at a present encounter, when the fighter has flying time $t$ remaining and $n$ missiles remaining. Three seemingly obvious properties of $K(n, t)$ have been conjectured: (A) the closer to the destination, the more of the $n$ missiles one should use; (B) the more missiles one has; the more one should use; and (C) the more missiles one has, the more one should save for possible future encounters. We show that (C) holds for all $0 \leq u \leq 1$, that (A) and (B) hold for the 'invincible fighter' $(u=1)$, and that (A) holds but (B) fails for the 'frail fighter' $(u=0)$; the latter is shown through a surprising counterexample, which is also valid for small $u>0$ values.
\end{abstract}

Keywords: Bomber problem; continuous ammunition; discrete ammunition; concavity

2010 Mathematics Subject Classification: Primary 60G40

Secondary 62L05

\section{Introduction}

We first describe two different models, the bomber and fighter models, with the following common probabilistic structure but with differing goals, stated below. In both models, there are two agents acting: an aircraft which is equipped with $n$ anti-aircraft missiles, and an enemy which sends its intercepting airplanes according to a Poisson process which, without loss of generality (by adjusting the time scale), we take to have intensity 1 . When meeting an enemy, one must decide how many of the $n$ available missiles one should 'spend' on it. The probability of destroying the enemy when attacking it with $j$ missiles is $a(j)$, where $a(0)=0$, and $\{a(j)\}$ is a strictly increasing and strictly concave sequence, for example, $a(j)=1-q^{j}$ for some $0<q<1$. This $\{a(j)\}$ corresponds to a situation where the missiles are independent and identically distributed Bernoulli with success probability $1-q$. If the aircraft fails to destroy the enemy, the enemy counterattacks it, and succeeds with a fixed, known probability $1-u$,

Received 29 July 2010; revision received 3 November 2010.

* Postal address: Department of Mathematics, University of Southern California, 3620 South Vermont Avenue, KAP 108, Los Angeles, CA 90089-2532, USA. Email address: bartroff@usc.edu

** Postal address: Department of Statistics and Center for the Study of Rationality, The Hebrew University of Jerusalem, Jerusalem, 91905, Israel. 
where $0 \leq u \leq 1$. Let $T>0$ and $[0, T]$ be a fixed time period. The bomber and fighter differ only in their goals.

Bomber: To maximize the probability of reaching the target $T$ safely.

Fighter: To maximize the number of enemy planes shot down during the time period $[0, T]$.

Let $K(n, t)$ be the optimal number of missiles that should be spent when meeting an enemy at a time $t$ from the destination with $n$ missiles at hand, referred to as state $(n, t)$. Clearly, $K(n, t)$ differs for the two models, but it may seem intuitively obvious, or at least reasonable, that, for both cases, $K(n, t)$ possesses the following three properties.

(A) $K(n, t)$ is nonincreasing in $t$ for fixed $n$.

(B) $K(n, t)$ is nondecreasing in $n$ for fixed $t$.

(C) $n-K(n, t)$ is nondecreasing in $n$ for fixed $t$.

The bomber problem with discrete ammunition was first considered in Klinger and Brown (1968). They assumed that (B) holds, and proved (A) under that assumption. Samuel (1970) proved (A) and (C) without any assumption about (B). The question of whether or not (B) holds for the bomber with discrete ammunition is still unsettled. The problem is also considered in Simons and Yao (1990). For continuous ammunition, both of the problems have recently been considered in Bartroff et al. (2010a), (2010b), and Bartroff (2011). In Bartroff et al. (2010a), (A) and (C) are shown to hold for the bomber problem with continuous ammunition, for which (B) is still an open problem. For the fighter with continuous ammunition, Bartroff et al. (2010a) showed that (A) and (C) hold for the frail fighter where $u=0$ (implying that if, at an encounter, the fighter fails to shoot down the enemy, he himself is shot down with probability 1 ), and that (B) and (C) hold for the invincible fighter where $u=1$, so he can never be shot down.

The fighter problem with an infinite time horizon and continuous ammunition $x$ was considered in Shepp et al. (1991), although the terminology there is different. In this case there is no dependence on $t$ and the optimal allocation can be denoted by $K(x)$. Shepp et al. showed that, for the frail case, where $u=0$, (B) fails.

In the present paper we consider the discrete ammunition case for the general fighter, $0 \leq$ $u \leq 1$. When not unique, we single out the optimal spending policy $K(n, t)$ which, at each stage, uses the smallest possible number of missiles; see (2.4). For this $K(n, t)$, we show in Section 3 that (C) holds for all $0 \leq u \leq 1$. In Section 4 we prove that both (A) and (B) hold for the invincible fighter, and we show that, for this case, $K(n, t)$ takes on all values $n, n-1, \ldots, 1$ as $t$ ranges from 0 to $\infty$. In Section 5 we prove that (A) holds for the frail fighter and show that (B) fails for $n=5$, and that it fails also for other small values of $u>0$. In Section 2 we introduce the necessary notation.

We apologize for using militaristic terminology, but we did not want to change the terminology already existing in the literature. We are convinced that the models are general enough to also have nonmilitaristic applications.

\section{The model}

Let $0=a(0)<a(1)<a(2)<\cdots$ be a concave sequence of probabilities, where $a(j)$ is the probability that the enemy will be shot down when $j$ missiles are shot at him. An example is $a(j)=1-q^{j}$, where $0<q<1$. Define the sequences $N_{n}(j, t)$ and $N(n, t)$ inductively as follows. Let $N_{n}(j, t)$ be the expected number of enemy airplanes shot down when confronting 
an enemy presently and spending $j$ out of the $n$ missiles available on it, and later continuing optimally. Let $N(n, t)$ be the total optimal expected number of enemy airplanes shot down when in state $(n, t)$, and confronting an enemy presently. Then

$$
N_{1}(1, t)=a(1)=N(1, t), \quad N(0, t)=0,
$$

and define $N_{n}(j, 0)=a(j)$ for $j=1, \ldots, n$. Let the present survival probability, when using $j$ missiles, be denoted by $c(j)$. Clearly,

$$
c(j)=a(j)+u(1-a(j))=a(j)(1-u)+u .
$$

Then

$$
N_{n}(j, t)=a(j)+c(j) N^{*}(n-j, t)
$$

where, for $r=1,2, \ldots$,

$$
N^{*}(r, t)=\int_{0}^{t} N(r, t-v) \mathrm{e}^{-v} \mathrm{~d} v=\mathrm{e}^{-t} \int_{0}^{t} N(r, x) \mathrm{e}^{x} \mathrm{~d} x=\mathrm{E} N(r, t-X),
$$

where $X$ has an exponential distribution and where we have set $N(r, t)=0$ for $t<0$. Then, inductively, we have

$$
N(n, t)=\max _{j=1, \ldots, n} N_{n}(j, t) .
$$

An optimal spending strategy is therefore

$$
K(n, t)=\min \left\{j: N_{n}(j, t)=\max _{i=1, \ldots, n} N_{n}(i, t)\right\} .
$$

We have written 'an optimal spending strategy' and not 'the optimal spending strategy' since the maximum in (2.4) may possibly be attained for more than one value of $j$, in which case we always choose the smallest, avoiding any issues of uniqueness and making $K(n, t)$ well defined. In what follows, $K(n, t)$ always refers to (2.4).

It follows from (2.2) that, for $j=1, \ldots, n$,

$$
N_{n+1}(j+1, t)=\frac{c(j+1)}{c(j)}\left[N_{n}(j, t)-a(j)\right]+a(j+1),
$$

which can also be written as

$$
N_{n}(j, t)=a(j)+\frac{c(j)}{c(j+1)}\left[N_{n+1}(j+1, t)-a(j+1)\right] .
$$

\section{Proof of (C)}

Lemma 3.1. For $j=1,2, \ldots$, let

$$
v(j)=a(j)-\frac{c(j)}{c(j+1)} a(j+1) .
$$

Then

$$
v(j) \leq v(j+1) \leq 0,
$$

and equality holds (in either or both places) if and only if $u=0$. 
Proof. When $u=0$, the equality in (3.1) is immediate, as $c(j)=a(j)$ in this case. Thus, assume that $u>0$. The right-hand inequality in (3.1) is immediate. Simplifying, it is easily seen that the other inequality in (3.1) can be written as

$$
\frac{a(j+1) c(j)-a(j) c(j+1)}{a(j+2) c(j+1)-a(j+1) c(j+2)} \geq \frac{c(j+1)}{c(j+2)} .
$$

Using (2.1), we see that the left-hand side of (3.2) simplifies to $[a(j+1)-a(j)] /[a(j+$ $2)-a(j+1)]$, and, thus, does not depend on $u$. Furthermore, by concavity, the value of the left-hand side is always greater than 1 , while the right-hand side of (3.2) depends on $u$ and is always less than, or equal to, 1. Thus, (3.2) holds.

We can rewrite (2.5) as

$$
N_{n}(j, t)=\frac{c(j)}{c(j+1)} N_{n+1}(j+1, t)+v(j),
$$

where $v(j) \leq 0$.

Theorem 3.1. Conjecture $(C)$ holds for the general fighter problem, i.e. for all $0 \leq u \leq 1$.

Proof. We must show that $n+1-K(n+1, t) \geq n-K(n, t)$, which can also be written as

$$
K(n+1, t) \leq K(n, t)+1 .
$$

Clearly, (3.4) holds when $K(n+1, t)=1$. Thus, assume that $K(n+1, t)=k$ for some $2 \leq k \leq n+1$ and some $t$. By (3.3), it follows that

$$
\begin{aligned}
N_{n}(j, t) & =\frac{c(j)}{c(j+1)} N_{n+1}(j+1, t)+v(j) \\
& \leq \frac{c(j)}{c(j+1)} N_{n+1}(k, t)+v(j) \\
& =\frac{c(j)}{c(j+1)} \frac{c(k)}{c(k-1)}\left[N_{n}(k-1, t)-v(k-1)\right]+v(j),
\end{aligned}
$$

where the inequality in (3.5) follows because $k$ is optimal for state $(n+1, t)$ and the last equality uses (3.3). Note that $k>1$ was used to avoid $k-1=0$. The maximum over $j=1, \ldots, n$ on the left-hand side of (3.5) is, by definition, attained for $j=K(n, t)$. Note, however, that, for all $j<k-1$, the value of the right-hand side of (3.5) is, by Lemma 3.1 and the concavity of the $a(j)$ sequence, less than $N_{n}(k-1, t)$. For $j=k-1$, equality holds throughout (3.5). Thus, the maximum over $j=1, \ldots, n$ of the left-hand side cannot be attained for any $j<k-1$, i.e. $K(n, t) \geq k-1$, which is (3.4).

A similar approach can be used to prove (C) for continuous ammunition for all $0 \leq u \leq 1$. In Bartroff et al. (2010b), (C) was shown to hold for only $u=0$ and $u=1$.

\section{Proofs of (A) and (B) for the invincible fighter}

We need the following lemma, valid for any $0 \leq u \leq 1$.

Lemma 4.1. If, for $n \geq 2$,

$$
N_{n}(j, t)-N_{n}(j+1, t) \text { is nondecreasing in } t \text { for } j=1, \ldots, n-1,
$$

then (A) holds for $n$. 
Proof. Suppose that the lemma is false. Then there exist values $t^{\prime}>t>0$ and $n \geq j>$ $r \geq 1$ such that $K\left(n, t^{\prime}\right)=j>K(n, t)=r$. But then

$$
N\left(n, t^{\prime}\right)=N_{n}\left(j, t^{\prime}\right)>N_{n}\left(r, t^{\prime}\right) \quad \text { and } \quad N(n, t)=N_{n}(r, t) \geq N_{n}(j, t) .
$$

Thus,

$$
\begin{aligned}
0 & \leq N_{n}(r, t)-N_{n}(j, t) \\
& =\sum_{i=r}^{j-1}\left[N_{n}(i, t)-N_{n}(i+1, t)\right] \\
& \leq \sum_{i=r}^{j-1}\left[N_{n}\left(i, t^{\prime}\right)-N_{n}\left(i+1, t^{\prime}\right)\right] \\
& =N_{n}\left(r, t^{\prime}\right)-N_{n}\left(j, t^{\prime}\right) \\
& <0,
\end{aligned}
$$

which is clearly a contradiction.

Note that, for the invincible fighter, (2.2) simplifies to

$$
N_{n}(j, t)=a(j)+N^{*}(n-j, t) .
$$

Lemma 4.2. For the invincible fighter and any $n \geq 2$, a sufficient condition for $N_{n}(j, t)-$ $N_{n}(j+1, t)$ to be strictly increasing in $t$, for $j=1, \ldots, n-1$, is that

$$
N(s, t)-N(s-1, t) \text { is nondecreasing in } t \text { for } s=1, \ldots, n-1 .
$$

Proof. By (4.1) and (2.3),

$$
\begin{aligned}
\frac{\mathrm{d}}{\mathrm{d} t}\left[N_{n}(j, t)-N_{n}(j+1, t)\right]= & \frac{\mathrm{d}}{\mathrm{d} t}\left[N^{*}(n-j, t)-N^{*}(n-j-1, t)\right] \\
= & {[N(n-j, t)-N(n-j-1, t)] } \\
& -\left[N^{*}(n-j, t)-N^{*}(n-j-1, t)\right] .
\end{aligned}
$$

Now if (4.2) holds,

$$
\begin{aligned}
N^{*}(n-j, t)-N^{*}(n-j-1, t) & =\mathrm{e}^{-t} \int_{0}^{t}[N(n-j, v)-N(n-j-1, v)] \mathrm{e}^{v} \mathrm{~d} v \\
& \leq \mathrm{e}^{-t}[N(n-j, t)-N(n-j-1, t)] \int_{0}^{t} \mathrm{e}^{v} \mathrm{~d} v \\
& =[N(n-j, t)-N(n-j-1, t)]\left(1-\mathrm{e}^{-t}\right) \\
& <N(n-j, t)-N(n-j-1, t),
\end{aligned}
$$

where the strict inequality follows as $N(s, t)-N(s-1, t)>0$ for all $s \geq 1$ and $t \geq 0$. Thus, the value in (4.3) is strictly positive.

It is important to note that (4.2) needs to hold for only $s$ up to $n-1$, as $j \geq 1$. Conjecture (A) will then hold for $n$, by Lemma 4.1. Also, note that, when (4.2) holds, $N^{*}(s, t)-N^{*}(s-1, t)$ is strictly increasing in $t$. 
Theorem 4.1. Conjecture (A) holds for the invincible fighter.

Proof. For $n=2$ and $s=1$ in (4.2), we have $N(1, t)-N(0, t)=a(1)$, which is nondecreasing in $t$; thus, (A) holds for $n=2$. For $s=2, N(2, t)-N(1, t)=\max \{a(2)-$ $\left.a(1), a(1)\left(1-\mathrm{e}^{-t}\right)\right\}$ and, as both of the expressions in the curly brackets are nondecreasing in $t$, it follows that (A) holds for $n=3$. (In our proof by induction we will need to go back two steps; thus, the need to show that (A) holds for $n=2$ and $n=3$ directly. A similar fact was overlooked in the proof in Samuel (1970).)

Now suppose that (4.2) holds for all $n \leq m$, where $m \geq 3$. We will show that it holds for $n=m+1$, i.e. we need to show that $N(m, t)-N(m-1, t)$ is nondecreasing in $t$. By the induction hypothesis we know that (A) holds for $n=m$ and $n=m-1$, and that $K(m, t)$ and $K(m-1, t)$ are constant on left-closed, right-open intervals. Suppose that in some interval of $t$-values $K(m, t)=j$ and $K(m-1, t)=r$. Then $1 \leq j \leq r+1$ by $(\mathrm{C})$, and, hence, in that interval

$$
\begin{aligned}
N(m, t)-N(m-1, t) & =N_{m}(j, t)-N_{m-1}(r, t) \\
& =N_{m}(j, t)-N_{m}(r+1, t)+a(r+1)-a(r),
\end{aligned}
$$

where the equality follows from (4.1). For $j=r+1$, the value in (4.4) is constant, and, hence, nondecreasing. For $1 \leq j \leq r$, write

$$
N_{m}(j, t)-N_{m}(r+1, t)=\sum_{i=j}^{r}\left[N_{m}(i, t)-N_{m}(i+1, t)\right],
$$

and note that all values in the square brackets in the sum are nondecreasing. This is a direct consequence of the induction hypothesis (which assumed that (4.2) holds for $n=m$ ) and Lemma 4.2. It follows that (4.2) holds for $n=m+1$ and, thus, by Lemma 4.1, that (A) holds for $n=m+1$.

We now show that (B) holds for the invincible fighter. The proof of the following lemma is similar to that of Lemma 4.1, and is thus omitted.

Lemma 4.3. Suppose that $N_{n}(j+1, t)-N_{n}(j, t)$ is nondecreasing in $n$ for $j=1, \ldots, n-1$ and fixed $t$. Then $K(n, t)$ is nondecreasing in $n$, i.e. $(B)$ holds.

Lemma 4.4. Suppose that $N(s, t)$ is (strictly) concave in $s$ for $s=0,1, \ldots, k$ and all fixed $t$. Then so is $N^{*}(s, t)$.

Proof. The result is immediate from (2.3).

The next two lemmas are slight modifications of Lemmas 3.1 and 5.1 of Bartroff et al. (2010a), the differences being (i) the domains of the functions here are nonnegative integers, and (ii) when the definition of $g(s)$ is for $s$ up to $k$, the resulting definition of $h(s)$ is for $s$ up to $k+1$. The proofs are omitted.

Lemma 4.5. Let $f(j), j=1,2, \ldots$, be a nonnegative log-concave sequence, and let $g(s)$, $s=0,1, \ldots, k$, be a nonnegative log-concave function. For any given $n, n=1,2, \ldots, k+1$, define

$$
h(n)=\max _{j=1, \ldots, n}\{f(j) g(n-j)\} .
$$

Then $h(n)$ is log-concave in $n$ for $n=1, \ldots, k+1$. 
Lemma 4.6. Let $f^{*}(j), j=1,2, \ldots$, be a nonnegative concave sequence, and let $g^{*}(s)$, $s=0,1, \ldots, k$, be nonnegative concave in $s$. Define

$$
h^{*}(n)=\max _{j=1, \ldots, n}\left\{f^{*}(j)+g^{*}(n-j)\right\} .
$$

Then $h^{*}(n)$ is concave in $n$ for $n=1, \ldots, k+1$.

Theorem 4.2. Conjecture (B) holds for the invincible fighter.

Proof. We will use Lemma 4.6 and induction on $k$. Let $f^{*}(j)=a(j)$ for $j=1,2, \ldots$, which is a nonnegative concave sequence, and let $g^{*}(n-j)=N^{*}(n-j, t)$, where $t$ is fixed. Then the resulting $h^{*}(n)$ will, by $(4.1)$, be $N(n, t)$. Since $N^{*}(0, t)=0$ and $N^{*}(1, t)=$ $a(1)\left(1-\mathrm{e}^{-t}\right)$, it follows that $g^{*}(s)$ is trivially nonnegative concave for $s=0$, 1, i.e. for $k=1$. The resulting $N(n, t)$ is therefore concave in $n$ for $n=1,2$. Now use Lemma 4.4 to imply that $N^{*}(s, t)$ is concave for $s$ up to 2, which, again by Lemma 4.6, implies that $N(s, t)$ is concave for $s$ up to 3, etc. The sequence $N(n, t)$ is therefore concave for all $n$ and fixed $t$, which, by Lemma 4.4, implies that the $N^{*}(n, t)$ sequence is concave. We will see that the condition of Lemma 4.3, and, hence, (B), hold. Fix $j$, and let $n>j$. Then

$$
N_{n}(j+1, t)-N_{n}(j, t)=a(j+1)-a(j)+N^{*}(n-j-1, t)-N^{*}(n-j, t),
$$

which is nondecreasing in $n$ by the concavity of the $N^{*}(n, t)$ sequence.

Next we show that, for the invincible fighter, $K(n, t)$ takes on all values $n, n-1, \ldots, 1$ as $t$ ranges from 0 to $\infty$. No similar result has been shown for the bomber problem.

For $s=1,2, \ldots$, let

$$
D^{*}(s, t)=N^{*}(s, t)-N^{*}(s-1, t) .
$$

Lemma 4.7. For the invincible fighter, for fixed $n$ and $j=1, \ldots, n-1$, the equation

$$
N_{n}(j+1, t)=N_{n}(j, t)
$$

has a unique solution, to be denoted by $t(n, j)$. For $t<t(n, j)$, the inequality $N_{n}(j+1, t)>$ $N_{n}(j, t)$ holds, whereas, for $t>t(n, j)$, we have $N_{n}(j+1, t)<N_{n}(j, t)$.

Proof. Equation (4.6) can be written as

$$
D^{*}(n-j, t)=a(j+1)-a(j) .
$$

In the proof of (A) we showed that $D^{*}(s, t)$ is strictly increasing in $t$. Thus, if we establish the existence of $t(n, j)$, the rest of the claim follows. We will show that

(a) for any $s, \lim _{t \rightarrow 0} D^{*}(s, t)=0$, and

(b) for any $s, \lim _{t \rightarrow \infty} D^{*}(s, t)=a(1)$.

Claim (a) is trivially true, as the domain of the integration defining $N^{*}(s, t)$ tends to 0 . For (b), fix $s$ and let $p(t)$ denote the probability of at least $s$ enemy planes in the time period $(0, t)$, i.e. $p(t)=\mathrm{e}^{-t} \sum_{i \geq s} t^{i} / i ! \rightarrow 1$ as $t \rightarrow \infty$. Then $N^{*}(s, t)$ is bounded below by the expected number obtained by spending one unit of ammunition on each of the first $s$ enemies; neglecting the number shot down when there are fewer than $s$ arrivals, this gives $N^{*}(s, t) \geq \operatorname{sa}(1) p(t) \rightarrow$ $s a(1)$ as $t \rightarrow \infty$. On the other hand, if the fighter somehow knew there would be at least $s$ 
arrivals, it follows from concavity of $a(j)$ that the optimal strategy in state $(s, t)$ would be to spend one unit on each of the first $s$ enemies. Bounding the number shot down when there are fewer than $s$ arrivals by $s$, we have $N^{*}(s, t) \leq s(1-p(t))+s a(1) p(t) \rightarrow s a(1)$ as $t \rightarrow \infty$, showing that $N^{*}(s, t) \rightarrow s a(1)$, and (b) now follows from (4.5). Now (a) and (b) guarantee the existence of a solution to (4.7) by continuity of $D^{*}(s, t)$ in $t$, and, by concavity, this implies that $0<a(j+1)-a(j)<a(1)$.

Theorem 4.3. For the invincible fighter, $K(n, t)$ takes all the values $n, n-1, \ldots, 1$ as $t$ ranges from 0 to $\infty$.

Proof. In the notation of Lemma 4.7 it suffices to show that $t(n, j+1)<t(n, j)$ for $j=0, \ldots, n-1$, as clearly then $K(n, t)=j+1$ for $t(n, j+1) \leq t<t(n, j)$, where we have let $t(n, n)=0$ and $t(n, 0)=\infty$. Actually, we will show the stronger conclusion that

$$
t(n, j+1)<t(n+1, j+1)<t(n, j) \text { for } j=0, \ldots, n-1,
$$

which shows that $K(n+1, t)$ will change exactly once in every interval of constancy of $K(n, t)$.

The first inequality in (4.8) follows using (4.7) and the fact that $D^{*}(s, t)$ is strictly decreasing in $s$ and $t$, the former by the strict concavity of the $N^{*}(s, t)$ sequence for every fixed $t$, shown in the proof of Theorem 4.2. The second inequality follows using (4.7) and the fact that the sequence $\{a(j)\}$ is strictly concave.

\section{Proof of (A) and a counterexample to (B) for the frail fighter}

The ideas of the proofs in the present section are similar to those of Section 4. We will need the following lemma, which is a slightly changed version of the lemma in Samuel (1970).

Lemma 5.1. Fix $n$, and assume that the $f(i, t)$ are positive continuous functions of $t$ for $i=1, \ldots, n$ such that $f(i, t) / f(i+1, t)$ are monotone nondecreasing in t for $i=1, \ldots, n-1$. Let

$$
K(n, t)=\min \left\{j: f(j, t)=\max _{i=1, \ldots, n} f(i, t)\right\} .
$$

Then $K(n, t)$ is nonincreasing in $t$ and right continuous, i.e. (A) holds for $n$.

We omit the proof, as it is exactly the same as that in Samuel (1970). (The statement there required that $f(i, t) / f(i+1, t)$ be strictly increasing, but this was not used in the proof.)

Theorem 5.1. Conjecture (A) holds for the frail fighter.

Proof. We would like to show, for $f(i, t)=N_{n}(i, t)$ of Lemma 5.1, that $N_{n}(i, t) / N_{n}(i+1, t)$ is nondecreasing in $t$ for $i=1, \ldots, n-1$. This is immediate for $i=n-1$ as, clearly, $N_{n}(n-1, t) / a(n)$ is nondecreasing in $t$. Thus, (A) holds for $n=2$ (and all $u$ ). For the frail fighter, (2.2) simplifies to

$$
N_{n}(i, t)=a(i)\left[1+N^{*}(n-i, t)\right], \quad i=1, \ldots, n .
$$

Thus, for the conditions of Lemma 5.1 to hold, we must show that

$$
\frac{1+N^{*}(n-i, t)}{1+N^{*}(n-i-1, t)} \quad \text { for } i=1, \ldots, n-2
$$

is nondecreasing in $t$. Since $i \geq 1$ and (5.1) is trivial for $i=n-1$, (5.1) would imply that (A) holds for $n$, where $n \geq 3$. We will show that $N^{*}(k, t)$ is totally positive of order 2 $\left(\mathrm{TP}_{2}\right.$ - see Karlin (1968)), i.e. that the ratio $N^{*}(k, t) / N^{*}(k-1, t)$ is nondecreasing in $t$ for $k=2, \ldots, n-1$. Since $N^{*}(k, t)$ is nondecreasing in both variables, it follows similarly to the 
proof of Lemma 3.5 of Bartroff et al. (2010a) that $1+N^{*}(k, t)$ is also $\mathrm{TP}_{2}$, i.e. that (5.1) holds. After some cancellation, it follows that

$$
N^{*}(k-1, t)^{2} \frac{\mathrm{d}}{\mathrm{d} t}\left(\frac{N^{*}(k, t)}{N^{*}(k-1, t)}\right)=N(k, t) N^{*}(k-1, t)-N(k-1, t) N^{*}(k, t),
$$

and we would like to show that the value on the right-hand side is nonnegative. Suppose that we show that $N(k, t) / N(k-1, t)$ is nondecreasing in $t$. Then we would have

$$
N(k, t) N(k-1, t-X) \geq N(k, t-X) N(k-1, t),
$$

where $X$ is exponentially distributed, and where we define $N(k, t)=0$ for $t<0$. Taking expectations over $X$ in (5.3), it follows that the right-hand side of (5.2) is nonnegative. Thus, it suffices to show that

$$
\frac{N(k, t)}{N(k-1, t)} \text { is nondecreasing in } t \text { for } k=2, \ldots, n-1
$$

for (A) to hold for $n$. For $k=2$, the claim is trivial; thus, (A) holds for $n=3$. Now suppose that (5.4) holds for $n=m$, where $m \geq 3$. We will show that it holds for $n=m+1$. Note that, by (5.4), the only additional requirement is to show that $N(m, t) / N(m-1, t)$ is nondecreasing in $t$. By the induction hypothesis we know that (A) holds for $m$ and $m-1$, and that $K(m, t)$ and $K(m-1, t)$ are constant on left-closed, right-open intervals. Suppose that in some interval $K(m, t)=j$ and $K(m-1, t)=r$. Then, by $(\mathrm{C}), 1 \leq j \leq r+1 \leq m$ and

$$
\frac{N(m, t)}{N(m-1, t)}=\frac{N_{m}(j, t)}{N_{m-1}(r, t)}=\frac{N_{m}(j, t)}{N_{m}(r+1, t)} \frac{a(r+1)}{a(r)},
$$

which is constant in $t$, and, hence, nondecreasing, when $j=r+1$.

For $j \leq r$, write

$$
\frac{N_{m}(j, t)}{N_{m}(r+1, t)}=\prod_{i=j}^{r} \frac{N_{m}(i, t)}{N_{m}(i+1, t)} .
$$

Now (5.4) holds for $n=m$ by the induction hypothesis and it follows that each term in the product (5.6) is nondecreasing in $t$, and, hence, the left-hand side of (5.5) is nondecreasing in $t$, implying that (A) holds for $n=m+1$.

Remark. Unlike Theorem 4.3, a similar statement fails to hold for the frail fighter. Even in the simple case $a(j)=1-q^{j}$ and $n=2$ it is easily seen that spending both missiles is preferable to spending one for any $t$ whenever $\frac{1}{2}<q \leq 1$. More generally, spending $n$ is preferable to spending $n-1$ for any $t$ whenever $q>\frac{1}{2}^{1 /(n-1)}$.

Assertion 5.1. Conjecture (B) fails for the frail fighter.

Proof. Since we have shown that (A) holds, the optimal $K(n, t)$ can be found by comparing $N_{n}(j+1, t)$ and $N_{n}(j, t)$ for $j=n-1, \ldots, 1$. For the frail fighter with $a(j)=1-q^{j}$ and $q=\frac{1}{2}$, and letting $\mathbf{1}(\cdot)$ denote an indicator function, we find that, for $0 \leq t<\infty$,

$$
\begin{aligned}
& K(2, t)=2, \\
& K(3, t)=3 \mathbf{1}\left(t<\log \left(\frac{3}{2}\right)\right)+2 \mathbf{1}\left(t \geq \log \left(\frac{3}{2}\right)\right), \\
& K(4, t)=4 \mathbf{1}\left(t<\log \left(\frac{7}{6}\right)\right)+3 \mathbf{1}\left(t \geq \log \left(\frac{7}{6}\right)\right),
\end{aligned}
$$


and, finally,

$$
\begin{aligned}
K(5, t)= & 5 \mathbf{1}\left(t<\log \left(\frac{15}{14}\right)\right)+4 \mathbf{1}\left(\log \left(\frac{15}{14}\right) \leq t<\log \left(\frac{3}{2}\right)\right) \\
& +3 \mathbf{1}\left(\log \left(\frac{3}{2}\right) \leq t<2.694 \ldots\right)+2 \mathbf{1}(t \geq 2.694 \ldots),
\end{aligned}
$$

where $2.694 \ldots$ is the numerical solution to

$$
\frac{3}{4}\left[\frac{17}{8}-\mathrm{e}^{-t}\left(\frac{5}{4}+\frac{3}{8}\left(t-\log \left(\frac{3}{2}\right)\right)\right)\right]=\frac{7}{8}\left[1+\frac{3}{4}\left(1-\mathrm{e}^{-t}\right)\right] .
$$

Thus, for $t>2.694 \ldots$, we have $K(4, t)=3$, while $K(5, t)=2$, violating (B). Note that, by continuity in $u$, it is clear that a similar result (with a slightly different cut-off point) will hold for sufficiently small $u>0$.

\section{Acknowledgements}

We would like to thank Larry Goldstein and Yosef Rinott for many helpful discussions on the fighter and bomber problems. Bartroff's work was supported in part by grant DMS-0907241 from the National Science Foundation and a Faculty-in-Residence grant from the Albert and Elaine Borchard Foundation.

\section{References}

Bartroff, J. (2011). A proof of the Bomber problem's spend-it-all conjecture. Sequent. Anal. 30, $52-57$.

Bartroff, J., Goldstein, L. And Samuel-Cahn, E. (2010a). The spend-it-all region and small time results for the continuous Bomber problem. Sequent. Anal. 29, 275-291.

Bartroff, J., Goldstein, L., Rinott, Y. and Samuel-Cahn, E. (2010b). On optimal allocation of a continuous resource using an iterative approach and total positivity. Adv. Appl. Prob. 42, 795-815.

KARLIN, S. (1968). Total Positivity, Vol. I. Stanford University Press.

Klinger, A. And Brown, T. A. (1968). Allocating unreliable units to random demands. In Stochastic Optimization and Control (Proc. Adv. Sem., Madison, 1967), ed. H. F. Karreman, John Wiley, New York, pp. 173-209.

SAmuel, E. (1970). On some problems in operations research. J. Appl. Prob. 7, 157-164.

Shepp, L. A., Simons, G. And Yao, Y.-C. (1991). On a problem of ammunition rationing. Adv. Appl. Prob. 23, $624-641$. Simons, G. ANd YaO, Y.-C. (1990). Some results on the bomber problem. Adv. Appl. Prob. 22, 412-432. 